\title{
Effectiveness of COVID-19 vaccines against SARS-CoV-2 variants of concern: a systematic
}

\section{review and meta-analysis}

\author{
Baoqi Zeng ${ }^{1}$, Le Gao ${ }^{2}$, Qingxin Zhou ${ }^{3}$, Kai Yu ${ }^{1}$, and Feng Sun ${ }^{4}$ \\ ${ }^{l}$ Department of Science and Education, Peking University Binhai Hospital, Tianjin, China \\ ${ }^{2}$ Department of Pharmacology and Pharmacy, The University of Hong Kong, Hong Kong, China \\ ${ }^{3}$ Tianjin Centers for Disease Control and Prevention, Tianjin, China \\ ${ }^{4}$ Department of Epidemiology and Biostatistics, School of Public Health, Peking University Health Science \\ Centre, Beijing, China
}

\section{Corresponding author: Kai Yu}

Email: wzxkjk@126.com; Tel: +86-22-65665880

Address: Department of Science and Education, Peking University Binhai Hospital, Tianjin, China.

\section{Alternative corresponding author: Feng Sun}

Email: sunfeng@bjmu.edu.cn; Tel: +86-10-82801108

Address: Department of Epidemiology and Biostatistics, School of Public Health, Peking University Health Science Centre, Beijing, China 


\section{ABSTRACT}

Background. It was urgent and necessary to synthesize the evidence for vaccine effectiveness (VE) against SARS-CoV-2 variants of concern (VOC). We conducted a systematic review and meta-analysis to provide a comprehensive overview of the effectiveness profile of COVID-19 vaccines against VOC.

Methods. Published and preprinted randomized controlled trials (RCTs), cohort studies, and case-control studies that evaluated the VE against VOC (Alpha, Beta, Gamma, or Delta) were searched until 31 August 2021. Pooled estimates and 95\% confidence intervals (CIs) were calculated using random-effects meta-analysis. VE was defined as (1-estimate).

Results. Seven RCTs (51,169 participants), 10 cohort studies (14,385,909 participants) and 16 case-control studies (734,607 cases) were included. Eight COVID-19 vaccines (mRNA-1273, BNT162b2, ChAdOx1, Ad26.COV2.S, NVX-CoV2373, BBV152, CoronaVac, and BBIBP-CorV) were included in this analysis. Full vaccination was effective against Alpha, Beta/Gamma, and Delta variants, with VE of $88.3 \%$ (95\% CI, 82.4-92.2), 70.7\% (95\% CI, 59.9-78.5), and 71.6\% (95\% CI, 64.1-77.4), respectively. But partial vaccination was less effective, with VE of 59.0\% (95\% CI, 51.3-65.5), 49.3\% (95\% CI, 33.0-61.6), and 52.6\% (95\% CI, 43.3-60.4), respectively. mRNA vaccines seemed to have higher VE against VOC over others, significant interactions ( $p_{\text {interaction }}<0.10$ ) were observed between VE and vaccine type (mRNA vaccines vs. non-mRNA vaccines).

Conclusions. Full vaccination of COVID-19 vaccines is highly effective against Alpha variant, and moderate effective against Beta/Gamma and Delta variants. Partial vaccination has less VE against VOC. mRNA vaccines seem to have higher VE against Alpha, Beta/Gamma, and Delta variants over others. 
Keywords. SARS-CoV-2; COVID-19; variants of concern; systematic review; vaccine effectiveness.

\section{INTRODUCTION}

Since emerging of coronavirus disease 2019 (COVID-19) caused by a novel severe acute respiratory syndrome coronavirus 2 (SARS-CoV-2) in December 2019, more than 225 million cases and 4.6 million deaths have been documented worldwide as of 15 September 2021 [1]. COVID-19 vaccines have been rapidly developed, and proved to be highly effective in multiple randomized clinical trials (RCTs)[2-5] and observational studies [6-8]. Most current vaccines used SARS-CoV-2 spike protein as the key antigenic target based on the originally identified Wuhan lineage virus [9]. The B.1.1.7 (Alpha) variant was first identified from genomic sequencing of samples obtained from COVID-19 patients which accounted for an expanding proportion of cases in England in late 2020 [10].Subsequently, the emergence of the B.1.351 (Beta) variant in South Africa and the P.1 (Gamma) variant in Brazil increased the COVID-19 pandemic. In December 2020, a novel SARS-CoV-2 variant, the B.1.617.2 (Delta) variant was first detected in India, causing a sharp increase in COVID-19 cases and deaths in India and surrounding countries [11].The emerging Alpha, Beta, Gamma, and Delta variants were classified as variants of concern (VOC), which were associated with the transmission increasing, more severe disease situation (e.g., increased hospitalizations or deaths), significant reduction in neutralization by antibodies generated during previous infection or vaccination, reduced effectiveness of treatments or vaccines, or diagnostic detection failures [12-17].The importance of vaccination programs and efficient public health measures will be increased if VOC have increased transmissibility or virulence.[18] It was urgent and necessary to synthesize evidence of the VE of COVID-19 vaccines against VOC. To our knowledge, there are some studies evaluating the VE of COVID-19 vaccines against VOC [19-22], but no relevant systematic review or meta-analysis has 
been published to date. Therefore, to gain insight in the VE of COVID-19 vaccines against VOC, we conducted a comprehensive systematic review and meta-analysis including both RCTs and observational studies. This review of the VE of COVID-19 vaccines against VOC will support global response on public health measures and vaccination programs timely and evidence based.

\section{METHODS}

\section{Data sources and searches}

We conducted this systematic review according to the Preferred Reporting Items for Systematic reviews and Meta-Analyses (PRISMA) guidelines [23], the protocol was registered on PROSPERO (CRD42021273986). We searched for literature published on PubMed, Embase, Cochrane Library, and the ClinicalTrials.gov website on or before 4 August 2021. Meanwhile, we also searched the medRxiv website to include eligible preprints in the last three months (from 4 May 2021 to 4 August 2021). Keywords including "COVID-19", "SARS-CoV-2", "vaccine", and "variant" were used to search, the detailed search strategy was shown in the Supplementary material. Additionally, we identified references by searching the reference lists of included studies and relevant reviews. Considering that the researches on COVID-19 vaccines were updated quickly, we researched literature before submission on 31 August 2021.

\section{Selection of studies}

We included randomized controlled trials (RCTs), cohort studies, and case-control studies that evaluated the efficacy or effectiveness of COVID-19 vaccines against VOC including B.1.1.7 (Alpha), B.1.351 (Beta), P.1 (Gamma), and B.1.617.2 (Delta). Studies enrolling general population or special 
populations (e.g., healthcare workers) aged 16 years or older were included. For studies that only reported VE against SARS-CoV-2 infections (without subgroup analysis of VOC), but the specific VOC accounted for $50 \%$ or more among positive tests, they were also included in the analysis. We excluded study protocols, editorials, comments, reviews, news, case reports, conference abstracts, animal studies, in vivo experiments, and analysis of antibody neutralization. Searches were limited to English articles. Preprints published in peer-reviewed journals were excluded. The primary outcome was the VE of full vaccination against VOC.

\section{Data extraction}

Two authors reviewed titles and abstracts independently to identify eligible studies that met pre-specified inclusion criteria and extracted data. When consensus was lacking, a third reviewer was consulted. The journal name, study type, study location, vaccine information, number of participants, characteristics of subjects, and outcomes were extracted from eligible studies. We extracted SARS-CoV-2 infection information if results on both SARS-CoV-2 infection and symptomatic infection were reported. The adjusted VE or estimates of effect size (relative risks, incidence rate ratios, or odds ratios) with corresponding $95 \%$ confidence intervals (CIs) was extracted with priority. The risk of bias of RCTs was assessed using the Cochrane Collaboration's tool [24, 25]. The risk of bias of cohort and case-control studies was assessed using the Newcastle-Ottawa scale (NOS) [26].The NOS contains 8 categories relating to methodological quality, with a maximum of 9 points. A total score of 7-9 points is considered of good quality, while a score of 4-6 points of moderate quality, and a score of 1-3 points of low quality. Two investigators reviewed the studies and judged the risk of bias. 


\section{Statistical analysis}

Pooled estimates and 95\% CIs were calculated using DerSimonian and Laird random-effects meta-analysis [27]. Summary VE was defined as (1-pooled estimate) $\times 100 \%$. We performed subgroup analysis stratifying by study design, vaccine type, participant, and publication. $\mathrm{P}$ for the difference was calculated using random-effects meta-regression, a difference between the estimates of these subgroups was considered significant if $\mathrm{p}_{\text {interaction }}<0.10$ [28]. Statistical heterogeneity between the studies was assessed with the $\chi^{2}$ test and the $I^{2}$ statistics. $I^{2}$ values of $25 \%, 50 \%$, and $75 \%$ have been suggested to be indicators of low, moderate, and high heterogeneity, respectively [29]. All the analysis were performed with STATA 14.

\section{RESULTS}

\section{Literature Rearch and Study characteristics}

This systematic literature search identified 2226 publications and 596 preprints, after excluding duplicates and irrelevant papers, 78 published reports and 25 preprints were evaluated in full text for eligibility (Supplementary Figure 1). Finally, 33 articles (23 publications [6, 7, 19-22, 30-46] and 10 preprints [47-56]) were included in the present systematic review. There were different study designs for included studies, 7 RCTs (51,169 participants), 10 cohort studies (14,385,909 participants), and 16 case-control studies $(734,607$ cases). In total, 8 COVID-19 vaccines (mRNA-1273, BNT162b2, ChAdOx1, Ad26.COV2.S, NVX-CoV2373, BBV152, CoronaVac and BBIBP-CorV) and 4 VOC (Alpha, Beta, Gamma and Delta) were included in this study. BNT162b2 and mRNA-1273 are mRNA vaccines, CoronaVac, BBV152 and BBIBP-CorV are inactivated vaccines, Ad26.COV2.S and 
ChAdOx1 are non-replicating vector vaccines, and NVX-CoV2373 is a protein subunit vaccine. Only Ad26.COV2.S is a single-dose vaccine, therefore a one-dose regimen is regarded as full vaccination. Characteristics of individual studies are summarized in Table 1.

\section{Risk of Bias}

All the RCTs were assessed as some concerns for overall risk-of-bias judgment. Seven of 10 cohort studies were judged as good quality, and the remaining 3 studies were moderate quality. For 16 case-control studies, 13 were considered as good quality and 3 were moderate quality. The detailed risk of bias assessment is available in online Supplementary Table 1-3.

\section{Vaccine Effectiveness of COVID-19 Vaccines against B.1.1.7 (Alpha) Variant}

Two RCTs [22, 44], 5 cohort studies [6, 7, 34, 38, 52] and 11 case-control studies [20, 30, 31, 33, 35, 39, 40, 43, 46, 47, 51] had evaluated the VE of COVID-19 vaccines against the Alpha variant. One cohort study [52] and 2 case-control studies [47, 51] were preprints on the medRxiv website. Four COVID-19 vaccines (mRNA-1273, NVX-CoV2373, ChAdOx1 and BNT162b2) were included in this analysis. Three studies enrolled healthcare workers [6, 33, 34], two enrolled participants aged 70 or older [30,35], and the others enrolled the general population. Characteristics of individual studies and VE for Alpha variant are summarized in Figure 1 and Supplementary Table 4.

The summary VE of full vaccination against the Alpha variant was 88.3\% (95\% CI, 82.4-92.2) (Table 2). Subgroup analysis by study design showed that VE was $79.4 \%(56.2-90.3)$ in 2 RCTs, $88.3 \%$ (95\% CI, 70.9-95.3) in 5 cohort studies and $88.7 \%$ (95\% CI, 84.2-92.0) in 10 case-control studies $\left(p_{\text {interaction }}=0.435\right)$. Subgroup analysis of vaccine type showed that VE was $90.9 \%$ (95\% CI, 86.2-94.0) 
for mRNA vaccines in 15 study groups, $73.8 \%$ (95\% CI, 69.8-77.4) for non-replicating vector vaccine in 5 study groups, $86.3 \%$ (95\% CI, 71.3-93.5) for protein subunit vaccine in 1 study group, and $82.0 \%$ (95\% CI, 27.0-95.0) for combined vaccines (BNT162b2/ChAdOx1) in 1 study group ( $\mathrm{p}_{\text {interaction }}=$ 0.084). And we detected a significant interaction $\left(\mathrm{p}_{\text {interaction }}=0.009\right)$ between $\mathrm{VE}$ and vaccine type (mRNA vaccines vs. non-mRNA vaccines), the VE of mRNA vaccines seemed to be higher than others. The results of subgroup analysis for participant and publication were in Table 2 .

The summary VE of partial vaccination against the Alpha variant (Table 2) was 59.0\% (95\% CI, 51.3-65.5). Subgroup analysis of study design showed that VE was 53.3\% (95\% CI, 40.0-63.7) in 4 cohort studies and 60.6\% (95\% CI, 51.4-68.1) in 10 case-control studies ( $\left.\mathrm{p}_{\text {interaction }}=0.565\right)$. Subgroup analysis by vaccine type showed that VE was $61.5 \%$ (95\% CI, 51.9-69.3) for mRNA vaccines in 14 study groups, $56.9 \%$ (95\% CI, 41.7-68.1) for non-replicating vector vaccine in 4 study groups, and $21.0 \%$ (95\% CI, -3.0-39.0) for combined vaccines (BNT162b2/ChAdOx1) in 1 study group ( pinteraction $_{\text {in }}$ $=0.687)$.

\section{Vaccine Effectiveness of COVID-19 Vaccines against B.1.351 (Beta) and P.1 (Gamma) Variants}

Four RCTs [21, 39, 45, 56], 1 cohort study [50], and 4 case-control studies [40, 43, 46, 47] had evaluated the VE of COVID-19 vaccines against the Beta variant. One cohort study [36] and 3 case-control studies [32, 35, 49] had evaluated the VE of COVID-19 vaccines against the Gamma variant. Both Beta and Gamma have N501Y and E484K mutations, 2 studies used a combined Beta/Gamma group because of insufficient specimens [31, 51]. One RCT [56] and 3 case-control studies $[31,49,51]$ were preprints on the medRxiv website. Six COVID-19 vaccines (mRNA-1273, BNT162b2, NVX-CoV2373, ChAdOx1, CoronaVac and Ad26.COV2.S) were included in this 
analysis. Single-dose Ad26.COV2.S was considered a full vaccination. For the study population, 1 study enrolled health care workers [49], 2 studies enrolled participants aged 70 or older [32, 35], 2 studies enrolled participants from long-term care homes [36, 50], and the others enrolled the general population. Characteristics of individual studies and VE for Beta and Gamma variants are summarized in Figure 2 and Supplementary Table 5.

The summary VE of full vaccination against Beta variant were 73.2\% (95\% CI, 61.0-81.5) (Table 2). Subgroup analysis by study design showed that VE for Beta variant was $70.2 \%$ (95\% CI, 18.8-89.1) in 4 RCTs, $80.2 \%$ (95\% CI, 71.1-86.4) in 4 case-control studies, and $49.0 \%$ (95\% CI, 14.0-69.0) in 1 cohort study $\left(\mathrm{p}_{\text {interaction }}=0.933\right)$. Subgroup analysis of vaccine type showed that VE was $80.3 \%$ (95\% CI, 69.7-87.1) for mRNA vaccines in 6 studies, $44.9 \%$ (95\% CI, -34.5-77.4) for non-replicating vector vaccines in 2 studies, and $51.1 \%$ (95\% CI, -0.6-76.2) for protein subunit vaccine in 1 study $\left(\mathrm{p}_{\text {interaction }}=0.214\right)$. The summary VE of partial vaccination against Beta variant was $41.9 \%(95 \%$ CI, 3.3-65.2) in four studies.

The summary VE of full vaccination against Gamma variant was $47.5 \%$ (95\% CI, 40.1-54.0) in 4 study groups, and partial vaccination against Gamma variant was $40.6 \%$ (95\% CI, -31.1-73.1) in 2 study groups (Table 2). When Beta/Gamma variant was treated as one group, the summary VE of full vaccination was 70.7\% (95\% CI, 59.9-78.5) in 15 study groups, and partial vaccination against Gamma variant was $49.3 \%$ (95\% CI, 33.0-61.6) in 10 study groups. Subgroup analysis of vaccine types showed that VE of full vaccination against Beta/Gamma was 78.3\% (95\% CI, 69.4-84.6) for mRNA vaccines in 10 study groups and $47.3 \%$ (95\% CI, 35.4-57.0) for non-mRNA vaccines in 5 study groups $\left(p_{\text {interaction }}=0.042\right.$ ), the VE for mRNA vaccines seemed to be higher than others. The results are in Table 2. 


\section{Vaccine Effectiveness of COVID-19 Vaccines against B.1.617.2 (Delta) Variant}

One RCT [48], 4 cohort studies [41, 42, 52, 54] and 7 case-control studies [20, 37, 39, 47, 51, 53, 55]

had evaluated the VE of COVID-19 vaccines against the Delta variant. Seven studies are preprints on the medRxiv website [47, 48, 51-55]. Six COVID-19 vaccines (mRNA-1273, BBV152, ChAdOx1, BNT162b2, CoronaVac and BBIBP-CorV) were included in this analysis. Two studies enrolled health care workers or frontline workers [41, 53], 1 study enrolled participants in the nursing home [42], and the others enrolled the general population. Characteristics of individual studies and VE for Delta variant are summarized in Figure 3 and Supplementary Table 6.

The summary VE of full vaccination against the Delta variant was $71.6 \%$ (95\% CI, 64.1-77.4) (Table 2). Subgroup analysis of study design showed that VE for Delta variant was $65.2 \%$ (95\% CI, 33.1-83.0) in 1 RCT, $74.8 \%$ (95\% CI, 65.3-81.8) in 7 case-control studies, and $66.2 \%$ (95\% CI, 51.3-76.5) in 4 cohort studies ( $p_{\text {interaction }}=0.256$ ). Subgroup analysis by vaccine type showed that VE was $75.6 \%$ (95\% CI, 64.9-83.1) for mRNA vaccines in 11 study groups, 64.4\% (95\% CI, 59.8-68.4) for non-replicating vector vaccine in 4 study groups, and 62.5\% (95\% CI, 37.7-77.5) for inactivated vaccines in 2 study groups $\left(p_{\text {interaction }}=0.124\right)$. An interaction $\left(p_{\text {interaction }}=0.097\right)$ between $\mathrm{VE}$ and vaccine type (mRNA vaccines vs. non-mRNA vaccines) was found, the VE for mRNA vaccines seemed to be higher than others.

The summary VE of partial vaccination against the Delta variant was 52.6\% (95\% CI, 43.3-60.4) (Table 2). Subgroup analysis for study design showed that VE for Delta variant was $59.5 \%$ (95\% CI, 44.1-70.7) in 1 cohort study, and $49.3 \%$ (95\% CI, 39.0-57.9) in 7 case-control studies ( $\mathrm{p}_{\text {interaction }}=$ 0.469). Subgroup analysis of vaccine type showed that VE was 60.5\% (95\% CI, 49.2-69.4) for 
mRNA vaccines in 9 study groups, 38.2\% (95\% CI, 24.6-49.3) for non-replicating vector vaccine in 5 study groups, and $13.8 \%(95 \% \mathrm{CI}, 60.2-54.8)$ for inactivated vaccines in 1 study group $\left(\mathrm{p}_{\text {interaction }}=\right.$ 0.038). The VE for mRNA vaccines seemed to be higher than others.

\section{DISCUSSION}

We conducted this systematic review and meta-analysis to synthesize evidence on the VE of COVID-19 vaccines against VOC during the pandemic. This study has four main findings. First, full vaccination of COVID-19 vaccines was effective against Alpha, Beta/Gamma and Delta variants, with the VE of $88.3 \%$ (95\% CI, 82.4-92.2), 70.7\% (95\% CI, 59.9-78.5) and 71.6\% (95\% CI, 64.1-77.4), respectively. Second, partial vaccination has lower VE against Alpha, Beta/Gamma and Delta variants, with the VE of 59.0\% (95\% CI, 51.3-65.5), 49.3\% (95\% CI, 33.0-61.6), 52.6\% (95\% CI, 43.3-60.4), respectively. Third, mRNA vaccines (BNT162b2 or mRNA-1273) seemed to have higher VE against VOC over other vaccines. Fourth, more evidence was needed to evaluate the VE of COVID-19 against the Gamma and Delta variants. To our knowledge, our study is the first comprehensive systematic review and meta-analysis to characterize the VE of COVID-19 vaccines against VOC.

The evidence for the Gamma variant was insufficient, only four studies were included $[32,35,36$, 49]. One study [36] enrolled residents or staff in long term care homes and two [32, 35] enrolled older adults aged 70 years and older, which reflected the VE against Gamma variant for elderly and frail people. The remaining one study evaluating the CoronaVac vaccine against the Gamma variant enrolled healthcare workers, but unmeasured confounding which led to downward bias in the VE estimate was reported [49].

The main results in this study were in consistent with a recent meta-analysis for neutralizing 
antibodies against SARS-CoV-2 variants, which showed that Alpha, Beta, Gamma and Delta variants significantly escaped natural-infection-mediated neutralization, with an average of 1.4-fold, 4.1-fold, 1.8-fold, and 3.2-fold reduction in live virus neutralization assay [57]. Despite the reduction in neutralization titers against Alpha variant, they remain robust, and there is no evidence of vaccine escape in one study [58]. Escape of Beta variant from neutralization by convalescent plasma and vaccine-induced sera was observed in some studies $[12,59,60]$. Although neutralization titers against Gamma variant are reduced, it is hoped that immunization with vaccines designed against parent strains will protect Gamma variant infection [61]. The Delta variant escapes neutralization by some antibodies that target the receptor-binding domain or N-terminal domain, the neutralization titers against Delta was three to five folds than Alpha variant when two-dose of the vaccine administrated.[14] This study also supports the two-dose vaccine regimen recommended by the FDA and EMA, which is consistent with an in-vitro study for SARS-CoV-2 variants of concern [62].

This review included 3 study designs evaluating 8 COVID-19 vaccines against 4 VOC in different populations. There is high heterogeneity between studies, and high statistical heterogeneity is also observed in most analysis. Other factors like the definition of outcomes (all SARS-CoV-2 or symptomatic infection), days after vaccination and participant's characteristics (e.g., age and race) may also contribute to the heterogeneity. Therefore, we mainly performed narrative descriptive synthesis.

This systematic review and meta-analysis has some limitations. First, $21 \%$ of studies (7 of 33) are nonrandomized. The imbalance between groups in observational studies is a concern, so potential selection bias may be existent. Second, 30\% of studies (10 of 33) are preprints and have not been certified by peer review. We may not identify errors in data analysis or reporting due to the lack of a 
rigorous vetting process. Third, although we performed qualitative analysis by different stratifications, heterogeneity was still high in most quantitative analysis. Forth, VE against hospitalization or death related to VOC is not included in our analysis. Finally, the evidence of COVID-19 vaccines against Gamma and Delta variants is not enough, more research is needed in the future.

\section{CONCLUSION}

Full vaccination of COVID-19 vaccines is highly effective against Alpha variant, and moderate effective against Beta/Gamma and Delta variant. Partial vaccination has less effectiveness against all VOC. Therefore, full vaccination is recommended against variants of SARS-CoV-2. mRNA vaccines (BNT162b2 or mRNA-1273) seem to have higher VE against Alpha, Beta/Gamma, or Delta over other vaccines, but more evidence is needed to confirm this finding.

\section{Notes}

\section{Author contributions.}

KY and FS conceived the study. BZ and KY designed the study. BZ, LG and QZ undertook the literature review and extracted the data. BZ and QZ coded the statistical analysis, figures, and appendix. BZ and LG interpreted the data and wrote the first draft of the manuscript. All authors reviewed and revised subsequent drafts and approved the final version.

\section{Financial support.}

No sources of funding are declared for this manuscript.

\section{Potential conflicts of interest.}

The authors declare that they have no conflicts of interest. 


\section{References}

1. World Health Organization. WHO coronavirus disease (COVID-19) dashboard. Available at: https://www.who.int. Accessed 15 September 2021.

2. Voysey M, Costa Clemens SA, Madhi SA, et al. Single-dose administration and the influence of the timing of the booster dose on immunogenicity and efficacy of ChAdOx1 nCoV-19 (AZD1222) vaccine: a pooled analysis of four randomised trials. Lancet 2021; 397(10277): $881-91$.

3. Baden LR, El Sahly HM, Essink B, et al. Efficacy and Safety of the mRNA-1273 SARS-CoV-2 Vaccine. New Engl J Med 2021; 384(5): 403-16.

4. Polack FP, Thomas SJ, Kitchin N, et al. Safety and Efficacy of the BNT162b2 mRNA Covid-19 Vaccine. New Engl J Med 2020; 383(27): 2603-15.

5. Logunov DY, Dolzhikova IV, Shcheblyakov DV, et al. Safety and efficacy of an rAd26 and rAd5 vector-based heterologous prime-boost COVID-19 vaccine: an interim analysis of a randomised controlled phase 3 trial in Russia. Lancet 2021; 397(10275): 671-81.

6. Hall VJ, Foulkes S, Saei A, et al. COVID-19 vaccine coverage in health-care workers in England and effectiveness of BNT162b2 mRNA vaccine against infection (SIREN): a prospective, multicentre, cohort study. Lancet 2021; 397(10286): 1725-35.

7. Dagan N, Barda N, Kepten E, et al. BNT162b2 mRNA Covid-19 Vaccine in a Nationwide Mass Vaccination Setting. New Engl J Med 2021; 384(15): 1412-23.

8. Thompson MG, Burgess JL, Naleway AL, et al. Interim Estimates of Vaccine Effectiveness of BNT162b2 and mRNA-1273 COVID-19 Vaccines in Preventing SARS-CoV-2 Infection 
Among Health Care Personnel, First Responders, and Other Essential and Frontline Workers -

Eight U.S. Locations, December 2020-March 2021. MMWR-Morbid Mortal W 2021; 70(13): 495-500.

9. Le TT, Cramer JP, Chen R, Mayhew S. Evolution of the COVID-19 vaccine development landscape. Nature reviews Drug discovery 2020; 19(10): 667-8.

10. Rambaut A, Loman N, Pybus O, et al. Preliminary genomic characterisation of an emergent SARS-CoV-2 lineage in the UK defined by a novel set of spike mutations. 2020. Available at: https://virological.org/t/preliminary-genomic-characterisation-of-an-emergent-sars-cov-2-line age-in-the-uk-defined-by-a-novel-set-of-spike-mutations/563. Accessed 15 September 2021.

11. European Centre for Disease Prevention and Control. Threat assessment brief: emergence of SARS-CoV-2 B.1.617 variants in India and situation in the EU/EEA. 2021. Available at: https://www.ecdc.europa.eu/en/publications-data/threat-assessment-emergence-sars-cov-2-b1 617-variants. Accessed 15 September 2021.

12. Hoffmann M, Arora P, Groß R, et al. SARS-CoV-2 variants B.1.351 and P.1 escape from neutralizing antibodies. Cell 2021; 184(9): 2384-93.e12.

13. Garcia-Beltran WF, Lam EC, St Denis K, et al. Multiple SARS-CoV-2 variants escape neutralization by vaccine-induced humoral immunity. Cell 2021; 184(9): 2372-83.e9.

14. Planas D, Veyer D, Baidaliuk A, et al. Reduced sensitivity of SARS-CoV-2 variant Delta to antibody neutralization. Nature $\mathbf{2 0 2 1}$.

15. Challen R, Brooks-Pollock E, Read JM, Dyson L, Tsaneva-Atanasova K, Danon L. Risk of mortality in patients infected with SARS-CoV-2 variant of concern 202012/1: matched cohort study. BMJ (Clinical research ed) 2021; 372: n579. 
16. Walensky RP, Walke HT, Fauci AS. SARS-CoV-2 Variants of Concern in the United States-Challenges and Opportunities. JAMA 2021; 325(11): 1037-8.

17. CDC. SARS-CoV-2 Variant Classifications and Definitions. Available at: https://www.cdc.gov/coronavirus/2019-ncov/variants/variant-info.html. Accessed 15 September 2021.

18. Krause PR, Fleming TR, Longini IM, et al. SARS-CoV-2 Variants and Vaccines. New Engl J Med 2021; 385(2): 179-86.

19. Shinde V, Bhikha S, Hoosain Z, et al. Efficacy of NVX-CoV2373 Covid-19 Vaccine against the B.1.351 Variant. New Engl J Med 2021; 384(20): 1899-909.

20. Lopez Bernal J, Andrews N, Gower C, et al. Effectiveness of Covid-19 Vaccines against the B.1.617.2 (Delta) Variant. New Engl J Med 2021.

21. Madhi SA, Baillie V, Cutland CL, et al. Efficacy of the ChAdOx1 nCoV-19 Covid-19 Vaccine against the B.1.351 Variant. New Engl J Med 2021; 385(7):585-94.

22. Emary KRW, Golubchik T, Aley PK, et al. Efficacy of ChAdOx1 nCoV-19 (AZD1222) vaccine against SARS-CoV-2 variant of concern 202012/01 (B.1.1.7): an exploratory analysis of a randomised controlled trial. Lancet 2021; 397(10282): 1351-62.

23. Moher D, Liberati A, Tetzlaff J, Altman DG. Preferred reporting items for systematic reviews and meta-analyses: the PRISMA statement. BMJ 2009; 339: b2535.

24. Higgins JP, Altman DG, Gøtzsche PC, et al. The Cochrane Collaboration's tool for assessing risk of bias in randomised trials. BMJ 2011; 343: d5928.

25. Sterne JAC, Savović J, Page MJ, et al. RoB 2: a revised tool for assessing risk of bias in randomised trials. BMJ 2019; 366: 14898. 
26. Wells G, Shea B, O'Connell D, et al. The Newcastle-Ottawa Scale (NOS) for Assessing the Quality of Nonrandomised Studies in Meta-Analyses. 2008. Available at: http://www.ohri.ca/programs/clinical epidemiology/oxford.asp. Accessed 15 September 2021.

27. DerSimonian R, Laird N. Meta-analysis in clinical trials. Control Clin Trials 1986; 7(3): $177-88$.

28. Richardson M, Garner P, Donegan S. Interpretation of subgroup analyses in systematic reviews: A tutorial. Clin Epidemiol Glob 2019; 7(2): 192-8.

29. Higgins JP, Thompson SG, Deeks JJ, Altman DG. Measuring inconsistency in meta-analyses. BMJ 2003; 327(7414): 557-60.

30. Bernal JL, Andrews N, Gower C, et al. Effectiveness of the Pfizer-BioNTech and Oxford-AstraZeneca vaccines on covid-19 related symptoms, hospital admissions, and mortality in older adults in England: Test negative case-control study. BMJ 2021; 373:n1088

31. Chung H, He S, Nasreen S, et al. Effectiveness of BNT162b2 and mRNA-1273 covid-19 vaccines against symptomatic SARS-CoV-2 infection and severe covid-19 outcomes in Ontario, Canada: test negative design study. BMJ 2021; 374: n1943.

32. Ranzani OT, Hitchings MDT, Dorion M, et al. Effectiveness of the CoronaVac vaccine in older adults during a gamma variant associated epidemic of covid-19 in Brazil: test negative case-control study. BMJ 2021; 374: n2015.

33. Carazo S, Talbot D, Boulianne N, et al. Single-dose mRNA vaccine effectiveness against SARS-CoV-2 in healthcare workers extending 16 weeks post-vaccination: a test-negative design from Quebec, Canada. Clin Infect Dis 2021. 
34. Lumley SF, Rodger G, Constantinides B, et al. An observational cohort study on the incidence of SARS-CoV-2 infection and B.1.1.7 variant infection in healthcare workers by antibody and vaccination status. Clin Infect Dis 2021.

35. Skowronski DM, Setayeshgar S, Zou M, et al. Single-dose mRNA vaccine effectiveness against SARS-CoV-2, including Alpha and Gamma variants: a test-negative design in adults 70 years and older in British Columbia, Canada. Clin Infect Dis 2021.

36. Williams C, Al-Bargash D, Macalintal C, et al. COVID-19 Outbreak Associated with a SARS-CoV-2 P.1 Lineage in a Long-Term Care Home after Implementation of a Vaccination Program - Ontario, April-May 2021. Clin Infect Dis 2021.

37. Li XN, Huang Y, Wang W, et al. Efficacy of inactivated SARS-CoV-2 vaccines against the Delta variant infection in Guangzhou: A test-negative case-control real-world study. Emerg Microbes Infec 2021: 1-32.

38. Haas EJ, Angulo FJ, McLaughlin JM, et al. Impact and effectiveness of mRNA BNT162b2 vaccine against SARS-CoV-2 infections and COVID-19 cases, hospitalisations, and deaths following a nationwide vaccination campaign in Israel: an observational study using national surveillance data. Lancet 2021; 397(10287): 1819-29.

39. Sheikh A, McMenamin J, Taylor B, Robertson C. SARS-CoV-2 Delta VOC in Scotland: demographics, risk of hospital admission, and vaccine effectiveness. Lancet 2021; 397(10293): 2461-2.

40. Charmet $\mathrm{T}$, Schaeffer L, Grant R, et al. Impact of original, B.1.1.7, and B.1.351/P.1 SARS-CoV-2 lineages on vaccine effectiveness of two doses of COVID-19 mRNA vaccines: Results from a nationwide case-control study in France. Lancet Reg Health Eur 2021; 8: 
100171.

41. Fowlkes A, Gaglani M, Groover K, Thiese MS, Tyner H, Ellingson K. Effectiveness of COVID-19 Vaccines in Preventing SARS-CoV-2 Infection Among Frontline Workers Before and During B.1.617.2 (Delta) Variant Predominance - Eight U.S. Locations, December 2020-August 2021. MMWR-Morbid Mortal W 2021; 70(34): 1167-9.

42. Nanduri S, Pilishvili T, Derado G, et al. Effectiveness of Pfizer-BioNTech and Moderna Vaccines in Preventing SARS-CoV-2 Infection Among Nursing Home Residents Before and During Widespread Circulation of the SARS-CoV-2 B.1.617.2 (Delta) Variant - National Healthcare Safety Network, March 1-August 1, 2021. MMWR-Morbid Mortal W 2021; 70(34): 1163-6.

43. Abu-Raddad LJ, Chemaitelly H, Butt AA. Effectiveness of the BNT162b2 Covid-19 Vaccine against the B.1.1.7 and B.1.351 Variants. New Engl J Med 2021; 385(2): 187-9.

44. Heath PT, Galiza EP, Baxter DN, et al. Safety and Efficacy of NVX-CoV2373 Covid-19 Vaccine. New Engl J Med 2021.

45. Sadoff J, Gray G, Vandebosch A, et al. Safety and Efficacy of Single-Dose Ad26.COV2.S Vaccine against Covid-19. New Engl J Med 2021; 384(23): 2187-201.

46. Chemaitelly H, Yassine HM, Benslimane FM, et al. mRNA-1273 COVID-19 vaccine effectiveness against the B.1.1.7 and B.1.351 variants and severe COVID-19 disease in Qatar. Nat Med 2021: 27(9):1614-1621

47. Chemaitelly $\mathrm{H}$, Tang $\mathrm{P}$, Hasan MR, et al. Waning of BNT162b2 vaccine protection against SARS-CoV-2 infection in Qatar. medRxiv 2021: 2021.08.25.21262584.

48. Ella R, Reddy S, Blackwelder W, et al. Efficacy, safety, and lot to lot immunogenicity of an 
inactivated SARS-CoV-2 vaccine (BBV152): a, double-blind, randomised, controlled phase 3 trial. medRxiv 2021: 2021.06.30.21259439.

49. Hitchings MDT, Ranzani OT, Scaramuzzini Torres MS, et al. Effectiveness of CoronaVac among healthcare workers in the setting of high SARS-CoV-2 Gamma variant transmission in Manaus, Brazil: A test-negative case-control study. medRxiv 2021: 2021.04.07.21255081.

50. Lefèvre B, Tondeur L, Madec Y, et al. Impact of B.1.351 (beta) SARS-CoV-2 variant on BNT162b2 mRNA vaccine effectiveness in long-term care facilities of eastern France: a retrospective cohort study. medRxiv 2021: 2021.07.28.21261285.

51. Nasreen S, Chung H, He S, et al. Effectiveness of COVID-19 vaccines against variants of concern in Ontario, Canada. medRxiv 2021: 2021.06.28.21259420.

52. Pouwels KB, Pritchard E, Matthews PC, et al. Impact of Delta on viral burden and vaccine effectiveness against new SARS-CoV-2 infections in the UK. medRxiv 2021: 2021.08.18.21262237.

53. Pramod S, Govindan D, Ramasubramani P, et al. Effectiveness of Covishield vaccine in preventing Covid-19 - A test-negative case-control study. medRxiv 2021: 2021.07.19.21260693.

54. Puranik A, Lenehan PJ, Silvert E, et al. Comparison of two highly-effective mRNA vaccines for COVID-19 during periods of Alpha and Delta variant prevalence. medRxiv 2021. 2021.08.06.21261707

55. Tang P, Hasan MR, Chemaitelly H, et al. BNT162b2 and mRNA-1273 COVID-19 vaccine effectiveness against the Delta (B.1.617.2) variant in Qatar. medRxiv 2021: 2021.08.11.21261885. 
56. Thomas SJ, Moreira ED, Kitchin N, et al. Six Month Safety and Efficacy of the BNT162b2 mRNA COVID-19 Vaccine. medRxiv 2021: 2021.07.28.21261159.

57. Chen X, Chen Z, Azman AS, et al. Neutralizing antibodies against SARS-CoV-2 variants induced by natural infection or vaccination: a systematic review and pooled meta-analysis. Clin Infect Dis 2021.

58. Supasa P, Zhou D, Dejnirattisai W, et al. Reduced neutralization of SARS-CoV-2 B.1.1.7 variant by convalescent and vaccine sera. Cell 2021; 184(8): 2201-11.e7.

59. Zhou D, Dejnirattisai W, Supasa P, et al. Evidence of escape of SARS-CoV-2 variant B.1.351 from natural and vaccine-induced sera. Cell 2021; 184(9): 2348-61.e6.

60. Li Q, Nie J, Wu J, et al. SARS-CoV-2 501Y.V2 variants lack higher infectivity but do have immune escape. Cell 2021; 184(9): 2362-71.e9.

61. Dejnirattisai W, Zhou D, Supasa P, et al. Antibody evasion by the P.1 strain of SARS-CoV-2. Cell 2021; 184(11): 2939-54.e9.

62. Skelly DT, Harding AC, Gilbert-Jaramillo J, et al. Two doses of SARS-CoV-2 vaccination induce robust immune responses to emerging SARS-CoV-2 variants of concern. Nat Commun 2021; $12(1): 5061$. 
Table 1 Study characteristics and participants demographics

\begin{tabular}{|c|c|c|c|c|c|c|c|}
\hline First Author & Journal & Study design & VOC & Vaccine & Country & Population Characteristics & Participants \\
\hline Shinde [19] & N Engl J Med & RCT (phase 2a/b) & Beta & NVX-CoV2373 & South Africa & GP; Age: $18-84$ & 4,387 \\
\hline Madhi [21] & N Engl J Med & RCT (phase 3) & Beta & ChAdOx1 & South Africa & GP; Age: 18-65 & 1,467 \\
\hline Heath [44] & N Engl J Med & RCT (phase 3) & Alpha & NVX-CoV2373 & UK & GP; Age: $18-84$ & 14,039 \\
\hline Sadoff [45] & N Engl J Med & RCT (phase 3) & Beta & Ad26.COV2.S & South Africa & GP; Age: $\geq 18$ & 4,969 \\
\hline Emary [22] & Lancet & RCT (phase 2/3) & Alpha & ChAdOx1 & UK & GP; Age: $\geq 18$ & 8,534 \\
\hline Ella [48] & Preprint & RCT (phase 3) & Delta & BBV152 & India & GP; Age: 18-98 & 16,973 \\
\hline Thomas [56] & Preprint & RCT (phase 2/3) & Beta & BNT162b2 & South Africa & GP; Age: $\geq 16$ & 800 \\
\hline Lopez Bernal [20] & N Engl J Med & TNCC & Alpha \& Delta & BNT162b2 or ChAdOx1 & UK & GP; Age: $\geq 16$ & 19,109 cases \\
\hline Abu-Raddad [43] & $\begin{array}{l}\text { N Engl J Med_ } \\
\text { Letter }\end{array}$ & TNCC & Alpha \& Beta & BNT162b2 & Qatar & GP; Age: $33(22-40)^{a}$ & 35,979 cases \\
\hline Sheikh [39] & $\begin{array}{l}\text { Lancet_- } \\
\text { Correspondence }\end{array}$ & TNCC & Alpha \& Delta & BNT162b2 or ChAdOx1 & UK & GP & 19,543 cases \\
\hline Chemaitelly [46] & Nat Med & TNCC & Alpha \& Beta & mRNA-1273 & Qatar & GP; Age: $32(25-39)^{a}$ & 66,042 cases \\
\hline Bernal [30] & BMJ & TNCC & Alpha & BNT162b2 & UK & Older adults; Age: $\geq 70$ & 3,034 cases \\
\hline Chung [31] & BMJ & TNCC & Alpha \& Beta/Gamma & BNT162b2 and mRNA-1273 & Canada & $\mathrm{GP} ; \geq 16$ & $\begin{array}{r}324,033 \\
\text { cases }\end{array}$ \\
\hline Ranzani [32] & BMJ & TNCC & Gamma & CoronaVac & Brazil & Older adults; $\geq 70$ & 43,774 cases \\
\hline Skowronski [35] & Clin Infect Dis & TNCC & Alpha \& Gamma & $\begin{array}{l}\text { BNT162b2 (85\%) and } \\
\text { mRNA-1273 (15\%) }\end{array}$ & Canada & Older adults; $\geq 70$ & 483 cases \\
\hline Carazo [33] & Clin Infect Dis & TNCC & Alpha & BNT162b2 and mRNA-1273 & Canada & HCWs; Age: 18-74 & 901 cases \\
\hline Li [37] & $\begin{array}{l}\text { Emerg Microbes } \\
\text { Infect }\end{array}$ & TNCC & Delta & CoronaVac and BBIBP-CorV & China & GP; Age: $18-59$ & 74 cases \\
\hline Charmet [40] & $\begin{array}{l}\text { Lancet Reg } \\
\text { Health Eur }\end{array}$ & Case-control & Alpha \& Beta/Gamma & BNT162b2 and mRNA-1273 & France & GP; Age: $\geq 20$ & 33,863 cases \\
\hline Nasreen [51] & Preprint & TNCC & Alpha \& Beta/Gamma & BNT162b2, mRNA-1273, or & Canada & GP; Age: $\geq 16$ & 40,828 cases \\
\hline
\end{tabular}




\begin{tabular}{|c|c|c|c|c|c|c|c|}
\hline & & & \& Delta & ChAdOx1 & & & \\
\hline Hitchings [49] & Preprint & TNCC & Gamma & CoronaVac & Brazil & HCWs; Age: $\geq 18$ & 418 cases \\
\hline Tang [55] & Preprint & TNCC & Delta & BNT $162 b 2$ or mRNA-1273 & Qatar & GP; Age: $31(24-37)^{\mathrm{a}}$ & 1,731 cases \\
\hline Chemaitelly [47] & Preprint & TNCC & Alpha \& Beta \& Delta & BNT162b2 & Qatar & GP; Age: $32(23-39)^{a}$ & $\begin{array}{r}144,075 \\
\text { cases }\end{array}$ \\
\hline Pramod [53] & Preprint & TNCC & Delta & ChAdOx1-S & India & HCWs; Age: $34(28-43)^{a}$ & 720 cases \\
\hline Hall [6] & Lancet & Prospective cohort & Alpha & $\begin{array}{l}\text { BNT162b2 (94\%) and } \\
\text { ChAdOx1 }(6 \%)\end{array}$ & UK & HCWs; Age: $\geq 18$ & 23,324 \\
\hline Haas [38] & Lancet & Retrospective cohort & Alpha & BNT162b2 & Israel & GP; Age: $\geq 16$ & $6,538,911$ \\
\hline Dagan [7] & N Engl J Med & Retrospective cohort & Alpha & BNT162b2 & Israel & GP; Age: $\geq 16$ & $1,193,236$ \\
\hline Lumley [34] & Clin Infect Dis & Retrospective cohort & Alpha & BNT162b2 and ChAdOx1 & UK & HCWs; Age: $39(30-50)^{\text {a }}$ & 13,109 \\
\hline Williams [36] & Clin Infect Dis & Retrospective cohort & Gamma & mRNA-1273 & Canada & Residents and staff of LTCH & 143 \\
\hline Nanduri [42] & $\begin{array}{l}\text { Morb Mortal } \\
\text { Wkly Rep }\end{array}$ & Retrospective cohort & Delta & mRNA-1273 or BNT162b2 & US & Nursing home residents & $5,965,607$ \\
\hline Fowlkes [41] & $\begin{array}{l}\text { Morb Mortal } \\
\text { Wkly Rep }\end{array}$ & Retrospective cohort & Delta & $\begin{array}{l}\text { mRNA-1273 }(33 \%) \text { and } \\
\text { BNT162b2 }(65 \%)\end{array}$ & US & Frontline workers & 2,840 \\
\hline Puranik [54] & Preprint & Retrospective cohort & Delta (July) & mRNA-1273 or BNT162b2 & US & GP; Age: $\geq 18$ & 67,469 \\
\hline Lefèvre [50] & Preprint & Retrospective cohort & Beta & BNT162b2 & France & $\begin{array}{l}\text { Residents of LTCH; } \\
\text { Age: } 89(83-92)^{a}\end{array}$ & 378 \\
\hline Pouwels [52] & Preprint & Retrospective cohort & Alpha \& Delta & $\begin{array}{l}\text { BNT162b2 or ChAdOx1 or } \\
\text { mRNA- } 1273\end{array}$ & UK & GP; Age: $\geq 18$ & 580,892 \\
\hline
\end{tabular}

Abbreviations: VOC, variants of concern; HCWs, healthcare workers; TNCC, Test-negative case-control; LTCH, long term care homes; GP, general population; RCT, randomized controlled trial.

${ }^{\mathrm{a}}$ Median age (interquartile range) 
Table 2. Meta-analysis and subgroup analysis for VE of COVID-19 against VOC

\begin{tabular}{|c|c|c|c|c|c|c|c|}
\hline Covariates & Subgroup & Study groups & Pooled estimates & $I^{2}$ & $\mathrm{p}(\mathrm{ES}=1)$ & $\mathrm{VE} \%(95 \% \mathrm{CI})$ & $\mathrm{p}_{\text {interaction }}$ \\
\hline \multicolumn{8}{|c|}{ Alpha (full vaccination) } \\
\hline \multicolumn{2}{|l|}{ All } & 22 & $0.117(0.078-0.176)$ & $98 \%$ & $<0.001$ & $88.3(82.4-92.2)$ & \\
\hline \multirow[t]{3}{*}{ Study design } & $\mathrm{RCT}$ & 2 & $0.206(0.097-0.438)$ & $58 \%$ & $<0.001$ & $79.4(56.2-90.3)$ & 0.435 \\
\hline & Case-control & 14 & $0.113(0.080-0.158)$ & $94 \%$ & $<0.001$ & $88.7(84.2-92.0)$ & \\
\hline & Cohort & 6 & $0.117(0.047-0.291)$ & $97 \%$ & $<0.001$ & $88.3(70.9-95.3)$ & \\
\hline \multirow[t]{6}{*}{ Vaccine type } & mRNA vaccines & 15 & $0.091(0.060-0.138)$ & $97 \%$ & $<0.001$ & $90.9(86.2-94.0)$ & 0.084 \\
\hline & Non-replicating vector vaccine & 5 & $0.262(0.226-0.302)$ & $0 \%$ & $<0.001$ & $73.8(69.8-77.4)$ & \\
\hline & Protein subunit vaccine & 1 & $0.137(0.065-0.287)$ & - & 0.012 & $86.3(71.3-93.5)$ & \\
\hline & mRNA vaccines or & & & & & & \\
\hline & Non-replicating vector vaccine & 1 & $0.180(0.050-0.730)$ & - & $<0.001$ & $82.0(27.0-95.0)$ & \\
\hline & Non-mRNA vaccines & 7 & $0.254(0.221-0.293)$ & - & $<0.001$ & $74.6(70.7-77.9)$ & $0.009^{\mathrm{a}}$ \\
\hline \multirow[t]{3}{*}{ Participant } & GP & 18 & $0.119(0.075-0.186)$ & $98 \%$ & $<0.001$ & $88.1(81.4-92.5)$ & 0.822 \\
\hline & HCWs & 3 & $0.096(0.058-0.158)$ & $8 \%$ & $<0.001$ & $90.4(84.2-94.2)$ & \\
\hline & Older & 1 & $0.100(0.061-0.163)$ & & $<0.001$ & $90.0(83.7-93.9)$ & \\
\hline \multirow[t]{2}{*}{ Publication } & Published & 15 & $0.101(0.063-0.163)$ & $98 \%$ & $<0.001$ & $89.9(83.7-93.7)$ & 0.143 \\
\hline & Preprint & 4 & $0.175(0.105-0.292)$ & $89 \%$ & $<0.001$ & $82.5(70.8-89.5)$ & \\
\hline \multicolumn{8}{|c|}{ Alpha (partial vaccination) } \\
\hline \multicolumn{2}{|l|}{ All } & 19 & $0.410(0.345-0.487)$ & $97 \%$ & $<0.001$ & $59.0(51.3-65.5)$ & \\
\hline \multirow[t]{2}{*}{ Study design } & Case-control & 14 & $0.394(0.319-0.486)$ & $98 \%$ & $<0.001$ & $60.6(51.4-68.1)$ & 0.565 \\
\hline & Cohort & 5 & $0.467(0.363-0.600)$ & $88 \%$ & 0.161 & $53.3(40.0-63.7)$ & \\
\hline \multirow[t]{3}{*}{ Vaccine type } & mRNA vaccines & 14 & $0.385(0.307-0.481)$ & $97 \%$ & $<0.001$ & $61.5(51.9-69.3)$ & 0.687 \\
\hline & $\begin{array}{l}\text { Non-replicating vector vaccine } \\
\text { mRNA vaccines or }\end{array}$ & 4 & $0.431(0.319-0.583)$ & $97 \%$ & $<0.001$ & $56.9(41.7-68.1)$ & \\
\hline & Non-replicating vector vaccine & 1 & $0.790(0.610-1.030)$ & - & 0.078 & $21.0(-3.0-39.0)$ & \\
\hline Participant & GP & 14 & $0.393(0.321-0.482)$ & $98 \%$ & $<0.001$ & $60.7(51.8-67.9)$ & 0.568 \\
\hline
\end{tabular}




\begin{tabular}{|c|c|c|c|c|c|c|c|}
\hline & HCWs & 3 & $0.461(0.268-0.793)$ & $91 \%$ & $<0.001$ & $53.9(20.7-73.2)$ & \\
\hline & Older & 2 & $0.463(0.243-0.883)$ & $94 \%$ & 0.019 & $53.7(11.7-75.7)$ & \\
\hline \multirow[t]{2}{*}{ Publication } & Published & 13 & $0.477(0.412-0.552)$ & $94 \%$ & $<0.001$ & $52.3(44.8-58.8)$ & 0.091 \\
\hline & Preprint & 6 & $0.306(0.242-0.388)$ & $93 \%$ & $<0.001$ & $69.4(61.2-75.8)$ & \\
\hline \multicolumn{8}{|c|}{ Beta (full vaccination) } \\
\hline \multicolumn{2}{|c|}{ All } & 9 & $0.268(0.185-0.390)$ & $85 \%$ & $<0.001$ & $73.2(61.0-81.5)$ & \\
\hline \multirow[t]{3}{*}{ Study design } & RCT & 4 & $0.298(0.109-0.812)$ & $85 \%$ & 0.018 & $70.2(18.8-89.1)$ & 0.933 \\
\hline & Case-control & 4 & $0.198(0.136-0.289)$ & $83 \%$ & $<0.001$ & $80.2(71.1-86.4)$ & \\
\hline & Cohort & 1 & $0.510(0.310-0.860)$ & $51 \%$ & 0.010 & $49.0(14.0-69.0)$ & \\
\hline \multirow[t]{3}{*}{ Vaccine type } & mRNA vaccines & 6 & $0.197(0.129-0.303)$ & $86 \%$ & $<0.001$ & $80.3(69.7-87.1)$ & 0.214 \\
\hline & Non-replicating vector vaccine & 2 & $0.551(0.226-1.345)$ & $77 \%$ & 0.191 & $44.9(-34.5-77.4)$ & \\
\hline & Protein subunit vaccine & 1 & $0.489(0.238-1.006)$ & - & 0.052 & $51.1(-0.6-76.2)$ & \\
\hline \multirow{2}{*}{ Participant } & GP & 8 & $0.246(0.166-0.365)$ & $85 \%$ & $<0.001$ & $75.4(63.5-83.4)$ & 0.524 \\
\hline & LTCH/Older & 1 & $0.510(0.310-0.860)$ & - & 0.010 & $49.0(14.0-69.0)$ & \\
\hline \multirow[t]{2}{*}{ Publication } & Published & 7 & $0.303(0.188-0.490)$ & $86 \%$ & $<0.001$ & $69.7(51.0-81.2)$ & 0.247 \\
\hline & Preprint & 2 & $0.059(0.002-1.395)$ & $91 \%$ & $\mathrm{p}=0.079$ & $94.1(-39.8-99.8)$ & \\
\hline \multicolumn{8}{|c|}{ Beta (partial vaccination) } \\
\hline \multicolumn{2}{|c|}{ All } & 4 & $0.581(0.348-0.967)$ & $98 \%$ & 0.037 & $41.9(3.3-65.2)$ & \\
\hline \multicolumn{8}{|c|}{ Gamma (full vaccination) } \\
\hline \multicolumn{2}{|l|}{ All } & 4 & $0.525(0.460-0.599)$ & $0 \%$ & $<0.001$ & $47.5(40.1-54.0)$ & \\
\hline \multicolumn{8}{|c|}{ Gamma (partial vaccination) } \\
\hline \multicolumn{2}{|l|}{ All } & 2 & $0.594(0.269-1.311)$ & $95 \%$ & 0.197 & $40.6(-31.1-73.1)$ & \\
\hline \multicolumn{8}{|c|}{ Beta/Gamma (full vaccination) } \\
\hline \multicolumn{2}{|c|}{ All } & 15 & $0.293(0.215-0.401)$ & $88 \%$ & $<0.001$ & 70.7 (59.9-78.5) & \\
\hline \multirow[t]{2}{*}{ Vaccine type } & mRNA vaccines & 10 & $0.217(0.154-0.306)$ & $81 \%$ & $<0.001$ & $78.3(69.4-84.6)$ & $0.042^{\mathrm{a}}$ \\
\hline & Non-mRNA vaccines & 5 & $0.527(0.430-0.646)$ & $15 \%$ & $<0.001$ & $47.3(35.4-57.0)$ & \\
\hline \multicolumn{8}{|c|}{ Beta/Gamma (partial vaccination) } \\
\hline \multicolumn{2}{|l|}{ All } & 10 & $0.507(0.384-0.670)$ & $96 \%$ & $<0.001$ & $49.3(33.0-61.6)$ & \\
\hline
\end{tabular}




\begin{tabular}{|c|c|c|c|c|c|c|c|}
\hline \multicolumn{8}{|c|}{ Delta (full vaccination) } \\
\hline \multicolumn{2}{|c|}{ All } & 17 & $0.284(0.226-0.359)$ & $94 \%$ & $<0.001$ & $71.6(64.1-77.4)$ & \\
\hline \multirow[t]{3}{*}{ Study design } & RCT & 1 & $0.348(0.170-0.669)$ & - & 0.003 & $65.2(33.1-83.0)$ & \multirow[t]{3}{*}{0.256} \\
\hline & Case-control & 10 & $0.252(0.182-0.347)$ & $94 \%$ & $<0.001$ & $74.8(65.3-81.8)$ & \\
\hline & Cohort & 6 & $0.338(0.235-0.487)$ & $95 \%$ & $<0.001$ & $66.2(51.3-76.5)$ & \\
\hline \multirow[t]{4}{*}{ Vaccine type } & mRNA vaccines & 11 & $0.244(0.169-0.351)$ & $96 \%$ & $<0.001$ & $75.6(64.9-83.1)$ & \multirow[t]{3}{*}{0.124} \\
\hline & Non-replicating vector vaccine & 4 & $0.356(0.316-0.402)$ & $42 \%$ & $<0.001$ & $64.4(59.8-68.4)$ & \\
\hline & Inactivated vaccine & 2 & $0.375(0.225-0.623)$ & $0 \%$ & $<0.001$ & $62.5(37.7-77.5)$ & \\
\hline & Non-mRNA vaccines & 6 & $0.354(0.323-0.387)$ & $7 \%$ & $<0.001$ & $64.6(61.3-67.7)$ & $0.097^{\mathrm{a}}$ \\
\hline \multirow[t]{3}{*}{ Participant } & GP & 14 & $0.261(0.206-0.332)$ & $92 \%$ & $<0.001$ & $73.9(66.8-79.4)$ & \multirow[t]{3}{*}{0.119} \\
\hline & Nursing home & 1 & $0.469(0.433-0.509)$ & - & $<0.001$ & $53.1(49.1-56.7)$ & \\
\hline & Frontline workers/HCWs & 2 & $0.424(0.286-0.630)$ & $0 \%$ & 0.006 & $57.6(37.0-71.4)$ & \\
\hline \multirow[t]{2}{*}{ Publication } & Published & 7 & $0.293(0.197-0.438)$ & $97 \%$ & $<0.001$ & $70.7(56.2-80.3)$ & \multirow[t]{2}{*}{0.841} \\
\hline & Preprint & 10 & $0.280(0.213-0.368)$ & $87 \%$ & $<0.001$ & $72.0(63.2-78.7)$ & \\
\hline \multicolumn{8}{|c|}{ Delta (partial vaccination) } \\
\hline All & Case-control, GP & 15 & $0.474(0.396-0.567)$ & $90 \%$ & $<0.001$ & $52.6(43.3-60.4)$ & \\
\hline \multirow[t]{2}{*}{ Study design } & Case-control & 12 & $0.507(0.421-0.610)$ & $87 \%$ & $<0.001$ & $49.3(39.0-57.9)$ & \multirow[t]{2}{*}{0.469} \\
\hline & Cohort & 3 & $0.405(0.293-0.559)$ & $85 \%$ & $<0.001$ & $59.5(44.1-70.7)$ & \\
\hline \multirow[t]{3}{*}{ Vaccine type } & mRNA vaccines & 9 & $0.395(0.306-0.508)$ & $87 \%$ & $<0.001$ & $60.5(49.2-69.4)$ & \multirow[t]{3}{*}{0.038} \\
\hline & Non-replicating vector vaccine & 5 & $0.618(0.507-0.754)$ & $86 \%$ & $<0.001$ & $38.2(24.6-49.3)$ & \\
\hline & Inactivated vaccine & 1 & $0.862(0.452-1.602)$ & - & 0.645 & $13.8(-60.2-54.8)$ & \\
\hline \multirow[t]{2}{*}{ Participant } & GP & 14 & $0.471(0.391-0.568)$ & $91 \%$ & $<0.001$ & $52.9(43.2-60.9)$ & \multirow[t]{2}{*}{0.839} \\
\hline & HCWs & 1 & $0.510(0.317-0.821)$ & - & 0.006 & $49.0(17.9-68.3)$ & \\
\hline \multirow[t]{2}{*}{ Publication } & Published & 5 & $0.727(0.659-0.801)$ & $55 \%$ & $<0.001$ & $27.3(19.9-34.1)$ & \multirow[t]{2}{*}{$<0.001$} \\
\hline & Preprint & 10 & $0.370(0.309-0.443)$ & $64 \%$ & $<0.001$ & $63.0(55.7-69.1)$ & \\
\hline
\end{tabular}

Abbreviations: VE, vaccine effectiveness; HCWs, healthcare workers; LTCH, long term care homes; GP, general population; RCT, randomized controlled trial; ES, effect size.

${ }^{a} \mathrm{P}$ for interaction between vaccine effectiveness and vaccine type (mRNA vaccines vs. non-mRNA vaccines). 


\section{Figure Legends}

Figure 1. Forest plot showing vaccine effectiveness of COVID-19 vaccines against Alpah variant. Abbreviations: VE, vaccine effectiveness; CI, confidence interval; RCT, randomized controlled trial.

Figure 2. Forest plot showing vaccine effectiveness of COVID-19 vaccines against

Beta/Gamma variants. Abbreviations: VE, vaccine effectiveness; CI, confidence interval;

RCT, randomized controlled trial.

Figure 3. Forest plot showing vaccine effectiveness of COVID-19 vaccines against Delta variant. Abbreviations: VE, vaccine effectiveness; CI, confidence interval; RCT, randomized controlled trial. 
Full vaccination

Chemaitelly (2021)

Haas (2021)

Lopez Bernal (2021)

Carazo (2021)

Dagan (2021)

Sheikh (2021)

Nasreen (preprint)

Chung (2021)

Bernal (2021)

Abu-Raddad (2021)

Nasreen (preprint)

Heath (2021)

Charmet (2021)

Hall (2021)

Lumley (2021)

Pouwels (preprint)

Pouwels (preprint)

Nasreen (preprint)

Lopez Bernal (2021)

Sheikh (2021)

Emary (2021)

Chemaitelly (preprint)

mRNA-1273_2D_14d

BNT162b2 2D 7d

BNT162b2_2D_14d

BNT162b2/mRNA-1273_2D_7d

BNT162b2_2D_14-7d

BNT162b2_2D_14d

mRNA-1273_2D_14d

BNT162b2/mRNA-1273_2D_7d

BNT162b2_2D_14d

BNT162b2_2D_14d

BNT162b2_2D_14d

NVX-CoV2373_2D_7d

BNT162b2/mRNA-1273_2D_7d

BNT162b2_2D_7d

BNT162b2/ChAdOx1_2D_14d

ChAdOx1_2D_14d

BNT162b2_2D_14d

ChAdOx1_2D_14d

ChAdOx1_2D_14d

ChAdOx1_2D_14d

ChAdOx1_2D_14d

BNT162b2_2D_0-4W

Partial vaccination

Chemaitelly (2021)

Nasreen (preprint)

Hall (2021)

Nasreen (preprint)

Nasreen (preprint)

Skowronski (2021)

Pouwels (preprint)

Chung (2021)

Carazo (2021)

Pouwels (preprint)

Chemaitelly (preprint)

Lopez Bernal (2021)

Lopez Bernal (2021)

Dagan (2021)

Sheikh (2021)

Sheikh (2021)

Bernal (2021)

Abu-Raddad (2021)

Lumley (2021)
mRNA-1273_1D_14d

BNT162b2_1D_21d

ChAdOx1_1D_21d

BNT162b2_1D_21d

BNT162b2/mRNA-1273 1D 21d

ChAdOx1_1D_21d

BNT162b2/mRNA-1273_1D_14d

BNT162b2/mRNA-1273_1D_14d

BNT162b2_1D_21d

BNT162b2_1D_14d

BNT162b2_1D_21d

BNT162b2_1D_14-21d

BNT162b2_1D_28d

ChAdOx1_1D_28d

BNT162b2 1D 21d

BNT162b2_1D_0d

BNT162b2/ChAdOx1_1D_14d
mRNA-1273_1D_21d

ChAdOx1_1D_21d
Case-control

Cohort

Case-control

Case-control

Cohort

Case-control

Case-control

Case-control

Case-control

Case-control

Case-control

RCT

Case-control

Cohort

Cohort

Cohort

Cohort

Case-control

Case-control

Case-control

RCT

Case-control

Case-control

Case-control

Cohort

Case-control

Case-control

Case-control

Cohort

Case-control

Case-control

Cohort

Case-control

Case-control

Case-control

Cohort

Case-control

Case-control

Case-control

Case-control

Cohort
$1.000(0.918,1.000)$

$0.965(0.963,0.968)$

$0.937(0.916,0.953)$

$0.926(0.871,0.958)$

$0.920(0.880,0.950)$

$0.920(0.900,0.930)$

$0.910(0.840,0.950)$

$0.900(0.850,0.940)$

$0.900(0.840,0.940)$

$0.895(0.859,0.923)$

$0.890(0.870,0.910)$

$0.863(0.713,0.935)$

$0.860(0.810,0.900)$

$0.860(0.760,0.970)$

$0.820(0.270,0.950)$

$0.790(0.560,0.900)$

$0.780(0.680,0.840)$

$0.750(-0.980,0.970)$

$0.745(0.684,0.794)$

$0.730(0.660,0.780)$

$0.704(0.436,0.845)$

$0.678(0.571,0.761)$

$0.881(0.837,0.915)$

$0.840(0.800,0.860)$

$0.720(0.580,0.860)$

$0.720(0.680,0.760)$

$0.690(0.670,0.710)$

$0.670(0.570,0.750)$

$0.630(0.550,0.690)$

$0.610(0.560,0.660)$

$0.600(0.536,0.655)$

$0.590(0.520,0.650)$

$0.549(0.280,0.724)$

$0.487(0.452,0.519)$

$0.475(0.416,0.528)$

$0.460(0.400,0.510)$

$0.380(0.290,0.450)$

$0.370(0.320,0.420)$

$0.362(0.237,0.466)$

$0.295(0.229,0.355)$

$0.210(-0.030,0.390)$ 
Full vaccination

Thomas (preprint)

Chemaitelly (2021)

Chung (2021)

Nasreen (preprint)

Charmet (2021)

Abu-Raddad (2021)

Chemaitelly (preprint)

Williams_staff (2021)

Sadoff (2021)

Williams_residents (2021)

Shinde (2021)

Lefèvre (2021)

Ranzani (2021)

Hitchings (preprint)

Madhi (2021)

Partial vaccination

Nasreen (preprint)

Nasreen (preprint)

Chemaitelly (2021)

Skowronski (2021)

Lefèvre (2021)

Nasreen (preprint)

Chung (2021)

Chemaitelly (preprint)

Abu-Raddad (2021)

Ranzani (2021)
BNT162b2_2D_7d

mRNA-1273_2D_14d

BNT162b2/mRNA-1273_2D_7d

BNT162b2_2D_14d

BNT162b2/mRNA-1273_2D_7d

BNT162b2_2D_14d

BNT162b2_2D_0-4W

mRNA-1273_2D_14d

Ad26.COV2.S_1D_28d

mRNA-1273_2D_14d

NVX-CoV2373_2D_7d

BNT162b2_2D_7d

CoronaVac_2D_14d

CoronaVac_2D_14d

ChAdOx1_2D_14d

mRNA-1273_1D_21d

BNT162b2_1D_21d

mRNA-1273_1D_14d

BNT162b2/mRNA-1273_1D_21d

BNT162b2_1D_14d

ChAdOx1_1D_21d

BNT162b2/mRNA-1273_1D_14d

BNT162b2_1D_14d

BNT162b2_1D_0d

CoronaVac_1D_14d

$\begin{array}{ll}\text { RCT } & \text { Beta } \\ \text { Case-control } & \text { Beta } \\ \text { Case-control } & \text { Beta/Gamma } \\ \text { Case-control } & \text { Beta/Gamma } \\ \text { Case-control } & \text { Beta } \\ \text { Case-control } & \text { Beta } \\ \text { Case-control } & \text { Beta } \\ \text { Cohort } & \text { Gamma } \\ \text { RCT } & \text { Beta } \\ \text { Cohort } & \text { Gamma } \\ \text { RCT } & \text { Beta } \\ \text { Cohort } & \text { Beta } \\ \text { Case-control } & \text { Gamma } \\ \text { Case-control } & \text { Gamma } \\ \text { RCT } & \text { Beta }\end{array}$

Case-control Beta/Gamma

Case-control Beta/Gamma

Case-control Beta

Case-control Gamma

Cohort Beta

Case-control Beta/Gamma

Case-control Beta/Gamma

Case-control Beta

Case-control Beta

Case-control Gamma

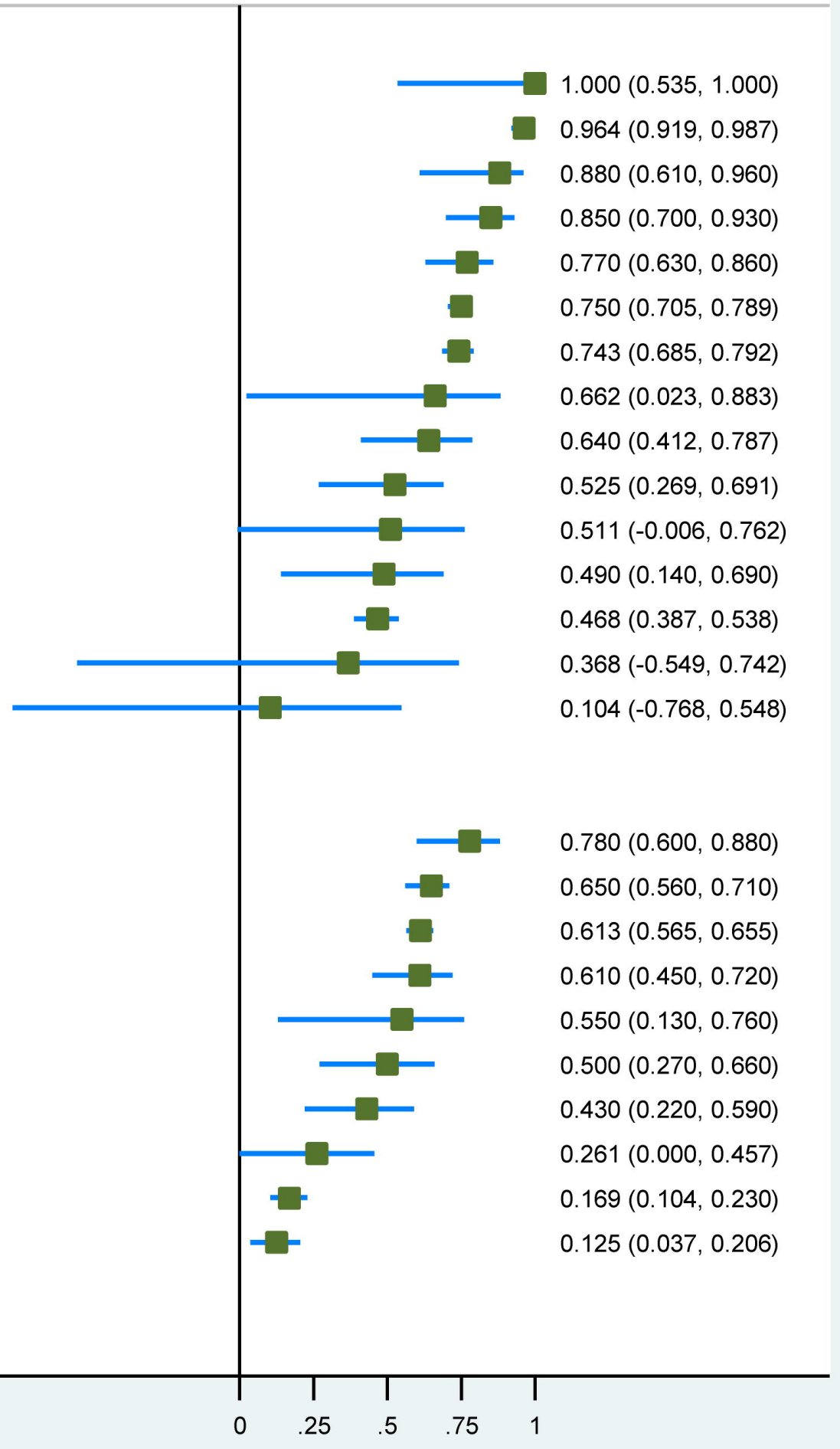


Full vaccination

Lopez Bernal (2021)

Tang (preprint)

BNT162b2 2D 14d

mRNA-1273_2D_14d

Nasreen (preprint)

BNT162b2_2D_14d

Chemaitelly (preprint) BNT162b2_2D_0-4W

Pouwels (preprint)

Sheikh (2021)

Puranik (preprint)

Lopez Bernal (2021)

Pouwels (preprint)

Fowlkes (2021)

Ella (preprint)

Sheikh (2021)

Tang (preprint)

Li (2021)

Pramod (preprint)

Nanduri (2021)

Nanduri (2021)

Puranik (preprint)

Partial vaccination

Tang (preprint)

Pouwels (preprint)

Nasreen (preprint)

Nasreen (preprint)

Chemaitelly (preprint)

Tang (preprint)

Nasreen (preprint)

Pouwels (preprint)

Pramod (preprint)

Pouwels (preprint)

Lopez Bernal (2021)

Lopez Bernal (2021)

Sheikh (2021)

Sheikh (2021)

Li (2021)
BNT162b2_2D_14d

BNT162b2_2D_14d

mRNA-1273 2D 14d

ChAdOx1_2D 14d

ChAdOx1_2D_14d

mRNA-1273/BNT162b2_2D_14d

BBV152 2D 14d

ChAdOx1_2D_14d

BNT162b2_2D_14d

CoronaVac/BBIBP-CorV_2D_14d

ChAdOx1-S_2D_14d

BNT162b2_2D_14d

mRNA-1273_2D_14d

BNT162b2_2D_14d

mRNA-1273_1D_14d

mRNA-1273_1D_21d

mRNA-1273_1D_21d

ChAdOx1 1D 21d

BNT162b2 1D_14d

BNT162b2_1D_14d

BNT162b2_1D_21d

BNT162b2 1D 21d

ChAdOx1-S_1D_21d

ChAdOx1_1D_21d

BNT162b2_1D_21d

ChAdOx1_1D_21d

BNT162b2_1D_28d

ChAdOx1_1D_28d

CoronaVac/BBIBP-CorV_1D_14d
Case-control

Case-control

Case-control

Case-control

Cohort

Case-control

Cohort

Case-control

Cohort

Cohort

RCT

Case-control

Case-control

Case-control

Case-control

Cohort

Cohort

Cohort

Case-control

Cohort

Case-control

Case-control

Case-control

Case-control

Case-control

Cohort

Case-control

Cohort

Case-control

Case-control

Case-control

Case-control

Case-control

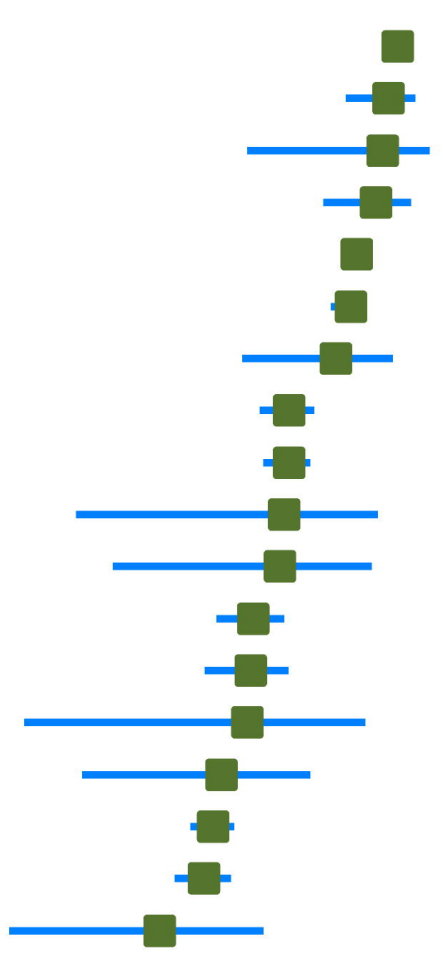

$0.880(0.853,0.901)$

$0.861(0.780,0.913)$

$0.850(0.590,0.940)$

$0.838(0.736,0.905)$

$0.800(0.770,0.830)$

$0.790(0.750,0.820)$

$0.760(0.580,0.870)$

$0.670(0.613,0.718)$

$0.670(0.620,0.710)$

$0.660(0.260,0.840)$

$0.652(0.331,0.830)$

$0.600(0.530,0.660)$

$0.596(0.507,0.669)$

$0.590(0.160,0.816)$

$0.540(0.270,0.710)$

$0.524(0.480,0.564)$

$0.506(0.450,0.557)$

$0.420(0.130,0.620)$

$0.797(0.608,0.895)$

$0.750(0.640,0.830)$

$0.700(0.520,0.810)$

$0.700(0.460,0.830)$

$0.674(0.463,0.809)$

$0.655(0.409,0.799)$

$0.610(0.510,0.700)$

$0.570(0.500,0.630)$

$0.490(0.170,0.680)$

$0.460(0.350,0.550)$

$0.356(0.227,0.464)$

$0.300(0.243,0.353)$

$0.300(0.170,0.410)$

$0.180(0.090,0.250)$

$0.138(-0.602,0.548)$ (1) 